\title{
Integral $p$-adic Hodge theory - announcement
}

\author{
B. Bhatt, M. Morrow and P. Scholze
}

\begin{abstract}
Given a proper, smooth (formal) scheme over the ring of integers of $\mathbb{C}_{p}$, we prove that if the crystalline cohomology of its special fibre is torsion-free then the $p$-adic étale cohomology of its generic fibre is also torsion-free. In this announcement we sketch the proof, which relies on the construction of a new cohomology theory interpolating crystalline and étale cohomology. Further details and results will be presented in the full forthcoming article.
\end{abstract}

\section{Introduction}

Let $C$ be an algebraically closed, nonarchimedean field of mixed characteristic which is complete under a rank one valuation; let $\mathcal{O} \subseteq C$ be its ring of integers, with maximal ideal $\mathfrak{m}$ and residue field $k$. The reader may assume that $C$ is the completion of an algebraic closure of $\mathbb{Q}_{p}$.

The main aim of this note is to outline a proof of the following result, which was first announced by the third author during his series of Fall 2014 lectures at the University of California, Berkeley. Details, generalisations, and further results, including more comparison isomorphisms than those given in Theorem 1.2, will be presented in a forthcoming article. ${ }^{1}$

Theorem 1.1. Let $\mathfrak{X}$ be a proper, smooth, formal scheme over $\mathcal{O}$, and let $i \geq 0$. Assume that the crystalline cohomology $H_{\text {crys }}^{i}\left(\mathfrak{X}_{k} / W(k)\right)$ of its special fibre $\mathfrak{X}_{k}$ is torsion-free. Then the p-adic étale cohomology $H_{\text {êt }}^{i}\left(X, \mathbb{Z}_{p}\right)$ of its generic fibre $X:=\mathfrak{X}_{C}$ is also torsion-free.

There are previous results of a similar flavour, at least in the case where $\mathfrak{X}$ is the $p$-adic completion of the base-change to $\mathcal{O}$ of a proper smooth scheme over a finite extension $V$ of $W(k)$, of ramification index $e$. Specifically, under stronger assumptions on torsion-freeness (for Hodge cohomology, and assuming degeneration of the mod $p$ Hodge-de Rham spectral sequence),

\footnotetext{
${ }^{1}$ Note added in proof: see [2].
} 
G. Faltings [7, Theorem 6] proves the result if $\operatorname{dim} X<p-1$. Moreover, $\mathrm{X}$. Caruso [4] proves that if $i e<p-1$, then the torsion in crystalline and in étale cohomology agree, which implies our theorem in that case. In the last section, we give an example showing that there may be torsion in crystalline cohomology while the étale cohomology is torsion-free; thus one cannot hope to extend Caruso's result to the general case.

As an example of our theorem which falls outside the scope of applicability of previous results, one may take for $\mathfrak{X}$ an Enriques surface over a 2-adic base. In that case, there is 2 -torsion in $H_{\text {ét }}^{2}\left(X, \mathbb{Z}_{2}\right)$, so that our theorem implies the existence of 2-torsion in $H_{\text {crys }}^{2}\left(\mathfrak{X}_{k} / W(k)\right)$, and thus non-vanishing of $H_{\mathrm{dR}}^{1}\left(\mathfrak{X}_{k} / k\right)$, which is a known pathology of Enriques surfaces in characteristic 2 [11, Proposition 7.3.8].

We note that the theorem may have applications to the study of torsion in the étale cohomology of Shimura varieties. For example, K.-W. Lan and J. Suh [12] prove first a vanishing result for torsion in crystalline cohomology, and then deduce the same for étale cohomology using existing results in integral $p$-adic Hodge theory.

Theorem 1.1 is proven by constructing a new cohomology theory valued in finitely presented $\mathbb{A}_{\text {inf }}$-modules (see $\S 2$ for a definition of $\mathbb{A}_{\text {inf }}$ and related objects) that interpolates the usual $p$-adic cohomology theories associated to $\mathfrak{X}$ :

Theorem 1.2. For any proper, smooth, formal scheme $\mathfrak{X}$ over $\mathcal{O}$, there is a perfect complex $R \Gamma_{\mathbb{A}}(\mathfrak{X})$ of $\mathbb{A}_{\mathrm{inf}}$-modules, functorial in $\mathfrak{X}$, with the following specializations:

- Étale specialization: $R \Gamma_{\mathbb{A}}(\mathfrak{X}) \otimes_{\mathbb{A}_{\text {inf }}}^{\mathbb{L}} W\left(C^{b}\right) \simeq R \Gamma_{\text {ét }}\left(X, \mathbb{Z}_{p}\right) \otimes_{\mathbb{Z}_{p}}^{\mathbb{L}} W\left(C^{b}\right)$.

- Crystalline specialization: $R \Gamma_{\mathbb{A}}(\mathfrak{X}) \otimes_{\mathbb{A}_{\text {inf }}}^{\mathbb{L}} W(k) \simeq R \Gamma_{\text {crys }}\left(\mathfrak{X}_{k} / W(k)\right)$.

- de Rham specialization: $R \Gamma_{\mathbb{A}}(\mathfrak{X}) \otimes_{\mathbb{A}_{\text {inf }}}^{\mathbb{L}} \simeq R \Gamma_{\mathrm{dR}}(\mathfrak{X} / \mathcal{O})$, where the base change is along Fontaine's map $\theta: \mathbb{A}_{\mathrm{inf}} \rightarrow \mathcal{O}$ (see §3.2).

Under the hypotheses of Theorem 1.1, the individual cohomology group $H_{\mathbb{A}}^{i}(\mathfrak{X}):=H^{i}(R \Gamma(\mathfrak{X}))$ enjoys similar specialization isomorphisms, from which the theorem follows; this will be explained in Section 2.

\section{Strategy of the proof}

Let $\mathcal{O}^{b}:=\lim _{\leftarrow} \mathcal{O} / p$ be the tilt (in the terminology of $[14, \S 3]$; classically $\mathcal{O}^{b}$ was denoted by $\left.\mathcal{R}_{\mathcal{O}}[9,1.2 .2]\right)$ of $\mathcal{O}$, whose field of fractions $C^{b}$ is an algebraically closed, nonarchimedean field of characteristic $p$, and let 
$\mathbb{A}_{\text {inf }}:=W\left(\mathcal{O}^{b}\right)$ be the first of Fontaine's period rings, on which we denote by $\varphi$ the usual Witt vector Frobenius. We will require the elements $\varepsilon:=\left(1, \zeta_{p}, \zeta_{p}, \ldots\right) \in \mathcal{O}^{b}$ and $\mu:=[\varepsilon]-1 \in \mathbb{A}_{\text {inf }}$, where $\zeta_{p}, \zeta_{p^{2}}, \cdots \in \mathcal{O}$ is a chosen compatible sequence of $p$-power roots of unity and [.] denotes the Teichmüller lifting. The results of our constructions will be independent of this chosen sequence of $p$-power roots of unity.

The theorems are proved by constructing a Zariski sheaf $\mathbb{A} \Omega_{\mathfrak{X} / \mathcal{O}}$ of complexes of $\mathbb{A}_{\text {inf }}$-modules on any smooth, formal $\mathcal{O}$-scheme $\mathfrak{X}$, satisfying the following properties:

$(\mathbb{A} 1) \mathbb{A} \Omega_{\mathfrak{X} / \mathcal{O}} \otimes_{\mathbb{A}_{\text {inf }}}^{\mathbb{L}} \mathbb{A}_{\text {inf }}\left[\frac{1}{\mu}\right] \simeq R \nu_{*} \mathbb{A}_{\text {inf }, X} \otimes_{\mathbb{A}_{\text {inf }}}^{\mathbb{L}} \mathbb{A}_{\text {inf }}\left[\frac{1}{\mu}\right]$, where $\nu$ is the projection from the pro-étale site of $X$ to the Zariski site of $\mathfrak{X}$ (see [15, §3] for the necessary theory of the pro-étale site of a rigid analytic space);

$(\mathbb{A} 2) \mathbb{A} \Omega_{\mathfrak{X} / \mathcal{O}} \otimes_{\mathbb{A}_{\text {inf }}}^{\mathbb{L}} W(k) \simeq W \Omega_{\mathfrak{X}_{k} / k}^{\bullet}$ after $p$-adic completion of the left-hand side;

(A3) $\mathbb{A} \Omega_{\mathfrak{X} / \mathcal{O}} \otimes_{\mathbb{A}_{\text {inf }}}^{\mathbb{L}} \mathcal{O} \simeq \Omega_{\mathfrak{X} / \mathcal{O}}^{\bullet}$, where the base change is along Fontaine's map;

$(\mathbb{A} 4) \mathbb{A} \Omega_{\mathfrak{X} / \mathcal{O}}$ is equipped with a $\varphi$-semilinear endomorphism $\varphi_{\mathbb{A}}$ which is compatible with $(\mathbb{A} 1)$ and $(\mathbb{A} 2)$.

Assuming that $\mathfrak{X}$ is proper, the cohomology theory of Theorem 1.2 is defined by $R \Gamma_{\mathbb{A}}(\mathfrak{X}):=R \Gamma\left(\mathfrak{X}, \mathbb{A} \Omega_{\mathfrak{X} / \mathcal{O}}\right)$. Now assume moreover that $H_{\text {crys }}^{i}\left(\mathfrak{X}_{k} / W(k)\right)$ is torsion-free. Then a Fitting ideal argument using $(\mathbb{A} 2)$ and $(\mathbb{A} 4)$ shows that the cohomology group $H_{\mathbb{A}}^{i}(\mathfrak{X})=H^{i}\left(\mathfrak{X}, \mathbb{A} \Omega_{\mathfrak{X} / \mathcal{O}}\right)$ is a finite free $\mathbb{A}_{\text {inf }}$-module equipped with a $\varphi$-semilinear endomorphism $\varphi_{\mathbb{A}}$ which becomes an isomorphism after inverting $\varphi(\xi)$, where $\xi:=\mu / \varphi^{-1}(\mu)$, in the sense that $\varphi_{\mathbb{A}}: \varphi^{*} H_{\mathbb{A}}^{i}(\mathfrak{X}) \otimes_{\mathbb{A}_{\text {inf }}} \mathbb{A}_{\text {inf }}\left[\frac{1}{\varphi(\xi)}\right] \stackrel{\widetilde{\Im}}{\rightarrow} H_{\mathbb{A}}^{i}(\mathfrak{X}) \otimes_{\mathbb{A}_{\text {inf }}} \mathbb{A}_{\text {inf }}\left[\frac{1}{\varphi(\xi)}\right]$; in other words, $H_{\mathbb{A}}^{i}(\mathfrak{X})$ is a Breuil-Kisin module in the sense of L. Fargues [8, §4].

Moreover, the Breuil-Kisin module $H_{\mathbb{A}}^{i}(\mathfrak{X})$ has the following étale and crystalline specializations, which follow respectively from $(\mathbb{A} 1)$ and $(\mathbb{A} 2)$ :

- $\left(H_{\mathbb{A}}^{i}(\mathfrak{X}) \otimes_{\mathbb{A}_{\text {inf }}} W\left(C^{b}\right)\right)^{\varphi_{\mathbb{A}}=1} \cong H_{\text {ét }}^{i}\left(X, \mathbb{Z}_{p}\right) ;$

- there is a canonical inclusion $H_{\mathbb{A}}^{i}(\mathfrak{X}) \otimes_{\mathbb{A}_{\text {inf }}} W(k) \hookrightarrow H_{\text {crys }}^{i}\left(\mathfrak{X}_{k} / W(k)\right)$ with torsion cokernel, which is an isomorphism if $H_{\text {crys }}^{i+1}\left(\mathfrak{X}_{k} / W(k)\right)$ is also assumed to be torsion-free.

Hence $H_{\text {ét }}^{i}\left(X, \mathbb{Z}_{p}\right)$ is a free $\mathbb{Z}_{p}$-module of the same rank as $H_{\text {crys }}^{i}\left(\mathfrak{X}_{k} / W(k)\right)$. Assuming the existence of the complex $\mathbb{A} \Omega_{\mathfrak{X} / \mathcal{O}}$, this completes the sketched 
proof of Theorem 1.1 and defines our new cohomology theory $H_{\mathbb{A}}^{i}(\mathfrak{X})$ interpolating the $p$-adic étale and crystalline cohomologies of the generic and special fibres of $\mathfrak{X}$ respectively.

Remark 2.1. When $\mathfrak{X}$ is the $p$-adic completion of a proper smooth $\mathcal{O}$ scheme, Theorem 1.1 follows more directly from the existence of $\mathbb{A} \Omega_{\mathfrak{X} / \mathcal{O}}$, at least if $H_{\text {crys }}^{i+1}\left(\mathfrak{X}_{k} / W(k)\right)$ is also torsion-free. Indeed, $R \Gamma_{\mathcal{O}^{b}}(\mathfrak{X}):=R \Gamma(\mathfrak{X}$, $\left.\mathbb{A} \Omega_{\mathfrak{X} / \mathcal{O}}\right) \otimes_{\mathbb{Z}} \mathbb{F}_{p}$ is a perfect complex of $\mathcal{O}^{b}$-modules, and perhaps the simplest new observable of our construction, whose fibres interpolate the étale and crystalline cohomology modulo $p$, thanks to $(\mathbb{A} 1)$ and $(\mathbb{A} 2)$ :

- $R \Gamma_{\mathcal{O}^{b}}(\mathfrak{X}) \otimes_{\mathcal{O}^{b}}^{\mathbb{L}} C^{b} \simeq R \Gamma_{\text {ét }}\left(X, \mathbb{F}_{p}\right) \otimes_{\mathbb{F}_{p}}^{\mathbb{L}} C^{b}$,

- $R \Gamma_{\mathcal{O}^{b}}(\mathfrak{X}) \otimes_{\mathcal{O}^{b}}^{\mathbb{L}} k \simeq R \Gamma_{\mathrm{dR}}\left(\mathfrak{X}_{k} / k\right)$.

By semicontinuity this gives $\operatorname{dim}_{\mathbb{F}_{p}} H_{\text {ét }}^{i}\left(X, \mathbb{F}_{p}\right) \leq \operatorname{dim}_{k} H_{\mathrm{dR}}^{i}\left(\mathfrak{X}_{k} / k\right)$, which yields the final of the following (in)equalities:

$$
\begin{aligned}
\operatorname{dim}_{W(k)\left[\frac{1}{p}\right]} H_{\text {crys }}^{i}\left(\mathfrak{X}_{k} / W(k)\right)\left[\frac{1}{p}\right] & =\operatorname{dim}_{\mathbb{Q}_{p}} H_{\text {ét }}^{i}\left(X, \mathbb{Q}_{p}\right) \\
& \leq \operatorname{dim}_{\mathbb{F}_{p}} H_{\text {ét }}^{i}\left(X, \mathbb{F}_{p}\right) \\
& \leq \operatorname{dim}_{k} H_{\mathrm{dR}}^{i}\left(\mathfrak{X}_{k} / k\right)
\end{aligned}
$$

Here the equality is a consequence of the crystalline comparison theorem and thus uses that $\mathfrak{X}$ is algebraic, while the first inequality is formal. If we now assume further that $H_{\text {crys }}^{*}\left(\mathfrak{X}_{k} / W(k)\right)$ for $*=i, i+1$ is torsion free, then the first and last dimensions in the above chain of (in)equalities are equal, and thus all the dimensions are equal. In particular,

$$
\operatorname{dim}_{\mathbb{Q}_{p}} H_{\text {ét }}^{i}\left(X, \mathbb{Q}_{p}\right)=\operatorname{dim}_{\mathbb{F}_{p}} H_{\text {ét }}^{i}\left(X, \mathbb{F}_{p}\right),
$$

which implies that $H_{\text {ét }}^{i}\left(X, \mathbb{Z}_{p}\right)$ is torsion free. (Note also that, by GAGA results $\left[10\right.$, Thm. 3.7.2], $H_{\text {ét }}^{i}\left(X, \mathbb{Z}_{p}\right)$ identifies with the $p$-adic étale cohomology of the generic fibre of the proper smooth $\mathcal{O}$-scheme of which $\mathfrak{X}$ is the $p$-adic completion.)

Remark 2.2. One can lift $(\mathbb{A} 2)$ above to a slightly larger portion of $\mathbb{A}_{\text {inf }}$ : the base change $\mathbb{A} \Omega_{\mathfrak{X} / \mathcal{O}} \otimes \mathbb{\mathbb { A }}_{\text {inf }} \mathbb{A}_{\text {crys }}$ can be identified with the (Zariski sheafified) crystalline cohomology of $\mathfrak{X}$ relative to $\mathbb{A}_{\text {crys }}$, where the latter is given the standard pd-structure. 


\section{Outline of construction of $\mathbb{A} \Omega_{\mathfrak{X} / \mathcal{O}}$}

It is sufficient to naturally (in an $\infty$-categorical sense) define the value of $\mathbb{A} \Omega_{\mathfrak{X} / \mathcal{O}}$ on arbitrary affine open formal subschemes of $\mathfrak{X}$, so we may assume that $\mathfrak{X}=\operatorname{Spf} R$, where $R$ is the $p$-adic completion of a smooth $\mathcal{O}$-algebra. Hence $X=\mathfrak{X}_{C}$ is the rigid analytic space $\operatorname{Spa}\left(R\left[\frac{1}{p}\right], R\right)$ over $C$, on whose pro-étale site we denote by $\mathbb{A}_{\inf , X}:=W\left(\lim _{\varphi} \mathcal{O}_{X}^{+} / p\right)$ the first period sheaf from [15, Def. 6.1]; note that $\mathbb{A}_{\text {inf }, X}$ is a sheaf of $\mathbb{A}_{\text {inf }}$-modules.

\subsection{Modifying torsion via the décalage functor $L \eta$}

We must first describe a general process for modifying torsion in complexes, based on P. Deligne's $[5,1.3 .3]$ décalé filtration and used previously by P. Berthelot and A. Ogus [1, Def. 8.6]. Let $A$ be a ring, $f \in A$ a non-zero divisor, and $N$ a cochain complex of $A$-modules supported in non-negative degrees such that $H^{0}(N)$ is $f$-torsion-free. Then we may replace $N$ by a quasi-isomorphic complex - still supported in non-negative degrees - consisting of $f$-torsion-free $A$-modules, and we then define $L \eta_{f} N$ to be the subcomplex of $N$ given by $L \eta_{f} N^{n}:=\left\{x \in f^{n} N^{n}: d x \in f^{n+1} N^{n+1}\right\}$ for $n \geq 0$. Then $L \eta_{f}: D_{\bar{f}-\mathrm{tf}}^{\geq 0}(A) \rightarrow D_{\bar{f}-\mathrm{tf}}^{\geq 0}(A)$ is a well-defined functor, where $D_{\bar{f}-\mathrm{tf}}^{\geq 0}(A)$ is the derived (or, more usefully, $\infty$-) category of complexes of $A$-modules supported in non-negative degrees with $f$-torsion-free $H^{0}$. Evidently there is a natural transformation $L \eta_{f} \rightarrow$ id.

Among various easily established identities (e.g., if $g \in A$ is another nonzero divisor then $L \eta_{f g}=L \eta_{f} L \eta_{g}$ and $^{2}\left(L \eta_{f}-\right) \otimes_{A}^{\mathbb{L}} A / g A \simeq L \eta_{f \bmod g A}\left(-\otimes_{A}^{\mathbb{L}}\right.$ $A / g A)$, under mild hypotheses), the most important is that there is a natural quasi-isomorphism

$$
L \eta_{f} N \otimes_{A}^{\mathbb{L}} A / f A \stackrel{\sim}{\rightarrow}\left(H^{\bullet}\left(N \otimes_{A}^{\mathbb{L}} A / f A\right), \text { Bock }\right),
$$

where the right side denotes the complex formed from the cohomology of $N \otimes_{A}^{\mathbb{L}} A / f A$ with differential given by the Bockstein operator $H^{n}\left(N \otimes_{A}^{\mathbb{L}}\right.$ $A / f A) \rightarrow H^{n+1}\left(N \otimes{ }_{A}^{\mathbb{L}} A / f A\right)$ associated to $f$.

\footnotetext{
${ }^{2}$ Note added in proof: the next identity is in fact false in general, but is proved by direct verification in the main paper in the cases of interest.
} 


\subsection{Constructing pro Witt complexes}

We now describe two general processes for building " $F$ - $V$-procomplexes over the $\mathcal{O}$-algebra $R$ " in the sense of Langer-Zink [13], among which their relative de Rham-Witt complex $\left\{W_{r} \Omega_{R / \mathcal{O}}^{\bullet}\right\}_{r}$ is the initial object. Note first that Fontaine's usual homomorphism of $p$-adic Hodge theory $\theta: \mathbb{A}_{\text {inf }} \rightarrow \mathcal{O}$, whose kernel is generated by the non-zero divisor $\xi$, lifts to a homomorphism $\theta_{r}: \mathbb{A}_{\text {inf }} \rightarrow W_{r}(\mathcal{O})$ whose kernel is generated by $\xi_{r}:=\mu / \varphi^{-r}(\mu)$; the Restriction $R$, Frobenius $F$, and Verschiebung $V$ on the Witt vectors $W_{r}(\mathcal{O})$ correspond via the homomorphisms $\theta_{r}$ respectively to id, $\varphi$, and $\xi \varphi^{-1}$ on $\mathbb{A}_{\text {inf }}$.

Let $D$ be a coconnective, commutative, differential graded (or, more generally, $\left.\mathbb{E}_{\infty^{-}}\right)$algebra over $\mathbb{A}_{\text {inf }}$ which is equipped with a $\varphi$-semilinear automorphism $\varphi_{D}$ and such that $H^{0}(D)$ is $\mu$-torsion-free. Assume also that there are isomorphisms of $W_{r}(\mathcal{O})$-algebras $\lambda_{r}: W_{r}(R) \stackrel{\widetilde{\simeq}}{\rightarrow} H^{0}\left(D \otimes \otimes_{\mathbb{A}_{\text {inf }}} \mathbb{A}_{\text {inf }} / \xi_{r}\right)$ for all $r \geq 1$ in such a way that the Restriction, Frobenius, and Verschiebung on the left correspond respectively to the canonical projection, $\varphi$, and $\xi \varphi^{-1}$ on the right.

First process: Equipping the cohomology groups $\mathcal{W}_{r}^{n}(D)_{\text {pre }}:=H^{n}\left(D \otimes_{\mathbb{A}_{\text {inf }}}^{\mathbb{L}}\right.$

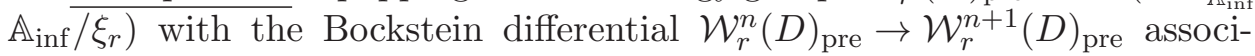
ated to $\xi_{r}$ makes $\mathcal{W}_{r}^{\bullet}(D)_{\text {pre }}$ into a commutative differential graded $W_{r}(\mathcal{O})$ algebra. Equipping them further with a Restriction, Frobenius, and Verschiebung given by " $\varphi^{-r}(\xi)^{n}$ times the canonical projection", $\varphi$, and $\xi \varphi^{-1}$ makes $\left\{\mathcal{W}_{r}^{\bullet}(D)_{\text {pre }}\right\}_{r}$ into an $F$ - $V$-procomplex over the $\mathcal{O}$-algebra $R$. (To be precise, the necessary identity that $F d \lambda_{r}([x])=\lambda_{r}\left(\left[x^{p-1}\right]\right) d \lambda_{r}([x])$ for all $x \in R$ does not appear to be automatic for arbitrary $D$, but will be satisfied in our cases of interest.) By the universal property of Langer-Zink's relative de Rham-Witt complex, there are then induced natural morphisms $\left\{W_{r} \Omega_{R / \mathcal{O}}^{\bullet}\right\}_{r} \rightarrow\left\{\mathcal{W}_{r}^{\bullet}(D)_{\text {pre }}\right\}_{r}$ of $F-V$-procomplexes over the $\mathcal{O}$-algebra $R$.

Improved process: It turns out that $\mathcal{W}_{r}^{\bullet}(D)_{\text {pre }}$ must be adjusted by some

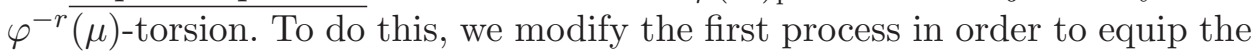
cohomology groups $\mathcal{W}_{r}^{\bullet}(D):=H^{\bullet}\left(L \eta_{\varphi^{-r}(\mu)} D \otimes_{\mathbb{A}_{\text {inf }}}^{\mathbb{A}} \mathbb{A}_{\text {inf }} / \xi_{r}\right)$ with the structure of an $F-V$-procomplex over the $\mathcal{O}$-algebra $R$. There are natural resulting morphisms

$$
\left\{W_{r} \Omega_{R / \mathcal{O}}^{\bullet}\right\}_{r} \longrightarrow\left\{\mathcal{W}_{r}^{\bullet}(D)\right\}_{r} \longrightarrow\left\{\mathcal{W}_{r}^{\bullet}(D)_{\text {pre }}\right\}_{r}
$$

of $F$ - $V$-procomplexes over the $\mathcal{O}$-algebra $R$.

The following is the key technical step in the construction of $\mathbb{A} \Omega_{R / \mathcal{O}}$ : 
Theorem 3.1. Apply the "Improved process" to $D=R \Gamma_{\text {proét }}\left(X, \mathbb{A}_{\text {inf }, X}\right)$. Then the induced map $W_{r} \Omega_{R / \mathcal{O}}^{\bullet} \rightarrow \mathcal{W}_{r}^{\bullet}\left(R \Gamma_{\text {proét }}\left(X, \mathbb{A}_{\text {inf }, X}\right)\right)$ descends to the p-adic completion of $W_{r} \Omega_{R / \mathcal{O}}^{\bullet}$, inducing an almost (wrt. the ideal $W_{r}(\mathfrak{m}) \subseteq$ $\left.W_{r}(\mathcal{O})\right)$ isomorphism of complexes of $W_{r}(\mathcal{O})$-modules for each $r \geq 1$ :

$$
\widehat{W_{r} \Omega_{R / \mathcal{O}}^{\bullet}} \stackrel{\text { al. }}{\stackrel{\sim}{\rightarrow}} \mathcal{W}_{r}^{\bullet}\left(R \Gamma_{\text {proét }}\left(X, \mathbb{A}_{\text {inf }, X}\right)\right)
$$

Proof. By a localisation and étale base change argument, the assertions reduce to the case that $R=\mathcal{O}\left\langle\underline{T}^{ \pm 1}\right\rangle:=\mathcal{O}\left\langle T_{1}^{ \pm 1}, \ldots, T_{d}^{ \pm 1}\right\rangle$. Let $\widetilde{R}:=\mathcal{O}\left\langle\underline{T}^{ \pm 1 / p^{\infty}}\right\rangle$, which is equipped with a continuous action of $\mathbb{Z}_{p}^{d}$ via $\mathcal{O}$-algebra homomorphisms, where the generator in the $i^{\text {th }}$-coordinate of $\mathbb{Z}_{p}^{d}$ acts via $T_{i}^{j / p^{k}} \mapsto$ $\zeta_{p^{k}}^{j} T_{i}^{j / p^{k}}$, for $j \in \mathbb{Z}$ and $k \geq 0$. In fact, $\widetilde{R}\left[\frac{1}{p}\right]$ is a perfectoid $C$-algebra occurring as the global sections of a pro-étale cover $\widetilde{X}:=“ \lim _{k}{ }_{k} \operatorname{Spa}\left(C\left\langle\underline{T}^{ \pm 1 / p^{k}}\right\rangle\right.$, $\left.\mathcal{O}\left\langle\underline{T}^{ \pm 1 / p^{k}}\right\rangle\right)$ of $X$, and there is a corresponding Hochschild-Serre map $R \Gamma_{\text {cont }}\left(\mathbb{Z}_{p}^{d}, H_{\text {proét }}^{0}\left(\widetilde{X}, \mathbb{A}_{\text {inf }, X}\right)\right) \rightarrow R \Gamma_{\text {proét }}\left(X, \mathbb{A}_{\text {inf }, X}\right)$. After tensoring by $\mathbb{A}_{\text {inf }} / \xi_{r} \cong W_{r}(\mathcal{O})$, this is an almost quasi-isomorphism (wrt. the ideal $\left.W_{r}(\mathfrak{m}) \subseteq W_{r}(\mathcal{O})\right)$ by Faltings' almost purity theorem [6, Thm. 3.1] [15, Thm. 6.5].

Applying the "Improved process" also to $D=R \Gamma_{\text {cont }}\left(\mathbb{Z}_{p}^{d}, H_{\text {proét }}^{0}(\widetilde{X}\right.$, $\left.\mathbb{A}_{\text {inf }, X}\right)$ ) we arrive at a commutative diagram of commutative, differential graded $W_{r}(\mathcal{O})$-algebras

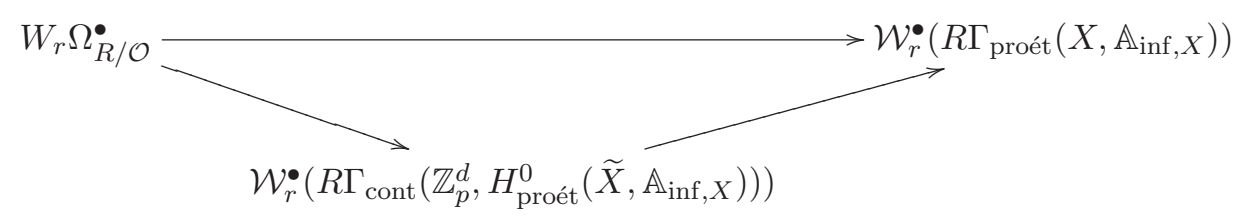

where the right diagonal arrow is an almost isomorphism by the final assertion of the previous paragraph. Up to explicit $\varphi^{-r}(\mu)$-torsion, the terms appearing in the complex at the bottom of the diagram are $H_{\text {cont }}^{\bullet}\left(\mathbb{Z}_{p}^{d}, W_{r}(\widetilde{R})\right)$; the top left of the diagram is even more explicit, using Langer-Zink's description of their relative de Rham-Witt complex in the case of a polynomial algebra $[13, \S 2]$. Using such explicit descriptions we verify directly that the diagonal left arrow becomes an isomorphism after $p$-adically completing $W_{r} \Omega_{R / \mathcal{O}}^{\bullet}$, and this completes the proof. 


\subsection{The final step: glueing}

From the definition of the improved $\mathcal{W}_{r}^{\bullet}(-)$-process and the stated properties of the $L \eta$ functor, there is a chain of quasi-isomorphisms

$$
\begin{aligned}
& \mathcal{W}_{r}^{\bullet}\left(R \Gamma_{\text {proét }}\left(X, \mathbb{A}_{\text {inf }, X}\right)\right) \\
= & \left(H^{\bullet}\left(L \eta_{\varphi^{-r}(\mu)} R \Gamma_{\text {proét }}\left(X, \mathbb{A}_{\text {inf }, X}\right) \otimes_{\mathbb{A}_{\text {inf }}}^{\mathbb{L}} \mathbb{A}_{\text {inf }} / \xi_{r}\right), \text { Bock }\right) \\
\simeq & L \eta_{\xi_{r}} L \eta_{\varphi^{-r}(\mu)} R \Gamma_{\text {proét }}\left(X, \mathbb{A}_{\text {inf }, X}\right) \otimes_{\mathbb{A}_{\text {inf }}}^{\mathbb{A}} \mathbb{A}_{\text {inf }} / \xi_{r} \\
\simeq & L \eta_{\mu} R \Gamma_{\text {proét }}\left(X, \mathbb{A}_{\text {inf }, X}\right) \otimes_{\mathbb{A}_{\text {inf }}}^{\mathbb{L}} \mathbb{A}_{\text {inf }} / \xi_{r},
\end{aligned}
$$

and hence the previous theorem yields an almost quasi-isomorphism

$$
\widehat{W_{r} \Omega_{R / \mathcal{O}}^{\bullet}} \stackrel{\text { al. }}{\simeq} L \eta_{\mu} R \Gamma_{\text {proét }}\left(X, \mathbb{A}_{\text {inf }, X}\right) \otimes_{\mathbb{A}_{\text {inf }}}^{\mathbb{L}} \mathbb{A}_{\text {inf }} / \xi_{r} .
$$

Let $R_{k}=R \otimes_{\mathcal{O}} k$. For each $r \geq 1$, we can reduce the preceding map from $\mathbb{A}_{\text {inf }} / \xi_{r} \cong W_{r}(\mathcal{O})$ to $W_{r}(k)$ to obtain the lower horizontal map in the diagram

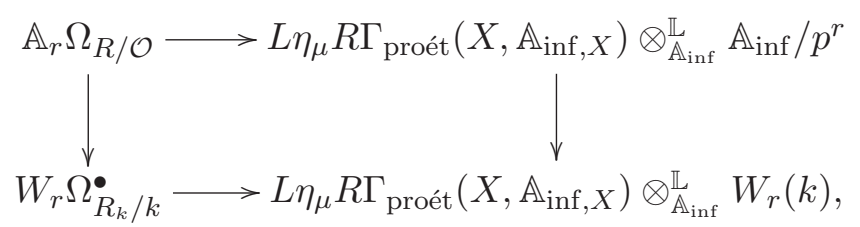

defining the top left complex $\mathbb{A}_{r} \Omega_{R / \mathcal{O}}$ of $\mathbb{A}_{\text {inf }} / p^{r}$-modules via (homotopy) pullback. As the lower two terms are almost zero, the upper horizontal arrow is an almost isomorphism. Moreover, the right vertical map is a quasiisomorphism after applying $-\otimes_{\mathbb{A}_{\text {inf }} / p^{r}}^{\mathbb{L}} W_{r}(k)$, since $W_{r}(k) \otimes_{\mathbb{A}_{\text {inf }} / p^{r}}^{\mathbb{L}} W_{r}(k) \simeq$ $W_{r}(k)$. This implies that there is a quasi-isomorphism

$$
\mathbb{A}_{r} \Omega_{R / \mathcal{O}} \otimes_{\mathbb{A}_{\text {inf }} / p^{r}}^{\mathbb{L}} W_{r}(k) \simeq W_{r} \Omega_{R_{k} / k}^{\bullet}
$$

We may now finally define

$$
\mathbb{A} \Omega_{R / \mathcal{O}}:=\operatorname{holim}_{r} \mathbb{A}_{r} \Omega_{R / \mathcal{O}}
$$

which is quasi-isomorphic, up to $W\left(\mathfrak{m}^{b}\right)$-torsion (we avoid the word "almost" here since $\left.W\left(\mathfrak{m}^{b}\right) \neq W\left(\mathfrak{m}^{b}\right)^{2}\right)$, to $L \eta_{\mu} R \Gamma\left(X, \mathbb{A}_{\text {inf }, X}\right)$ and satisfies $\mathbb{A} \Omega_{R / \mathcal{O}} \otimes_{\mathbb{A}_{\text {inf }}}^{\mathbb{L}}$ $W(k) \simeq W \Omega_{R_{k} / k}^{\bullet}$ after $p$-adic completion of the left-hand side. Then $\mathbb{A} \Omega_{R / \mathcal{O}}$ is equipped with a $\varphi$-semilinear operator $\varphi_{\mathbb{A}}$, by glueing those on $W \Omega_{R_{k} / k}^{\bullet}$ and $L \eta_{\mu} R \Gamma_{\text {proét }}\left(X, \mathbb{A}_{\text {inf }, X}\right)$. 
Since $\mu \in W\left(\mathfrak{m}^{b}\right)$, we have

$$
\begin{aligned}
\mathbb{A} \Omega_{R / \mathcal{O}} \otimes_{\mathbb{A}_{\text {inf }}}^{\mathbb{L}} \mathbb{A}_{\text {inf }}\left[\frac{1}{\mu}\right] & \simeq L \eta_{\mu} R \Gamma_{\text {proét }}\left(X, \mathbb{A}_{\text {inf }, X}\right) \otimes_{\mathbb{A}_{\text {inf }}}^{\mathbb{L}} \mathbb{A}_{\text {inf }}\left[\frac{1}{\mu}\right] \\
& \simeq R \Gamma_{\text {proét }}\left(X, \mathbb{A}_{\text {inf }, X}\right) \otimes_{\mathbb{A}_{\text {inf }}}^{\mathbb{L}} \mathbb{A}_{\text {inf }}\left[\frac{1}{\mu}\right]
\end{aligned}
$$

verifying $(\mathbb{A} 1)$. We have already verified $(\mathbb{A} 2)$; we omit the proof of $(\mathbb{A} 3)$ since it is not required for Theorem 1.1. This completes the outline of the construction of $\mathbb{A} \Omega_{\mathfrak{X} / \mathcal{O}}$.

\section{4. q-de Rham complexes}

An alternative perspective on $\mathbb{A} \Omega_{\mathfrak{X} / \mathcal{O}}$ and its construction is offered by the idea of $q$-de Rham complexes.

If $A$ is a ring, $q \in A^{\times}$, and $U$ is a formal variable, then the " $q$-de Rham complex" $q-\Omega_{A\left[U^{ \pm 1}\right] / A}^{\bullet}$ of the Laurent polynomial algebra $A\left[U^{ \pm 1}\right]$ is defined to be

$$
A\left[U^{ \pm 1}\right] \longrightarrow A\left[U^{ \pm 1}\right] \operatorname{dlog} U, \quad U^{j} \mapsto[j]_{q} U^{j} \operatorname{dlog} U
$$

where $\operatorname{d} \log U$ is a formal symbol and $[j]_{q}:=\frac{q^{j}-1}{q-1}$ is the " $q$-analogue of the integer $j$ ". In the more general case of a Laurent polynomial algebra in several variables $U_{1}, \ldots, U_{d}$, set

$$
q-\Omega_{A\left[U_{1}^{ \pm 1}, \ldots, U_{d}^{ \pm 1}\right] / A}^{\bullet}:=\bigotimes_{i=1}^{m} q-\Omega_{A\left[U_{i}^{ \pm 1}\right] / A}^{\bullet}
$$

Note that if $q=1$ then the $q$-de Rham complex equals the usual de Rham complex; more generally, it is a deformation of the de Rham complex over $\bar{A}:=A /(q-1)$, in the sense that $q-\Omega_{A\left[U_{1}^{ \pm 1}, \ldots, U_{d}^{ \pm 1}\right] / A}^{\bullet} \otimes_{A} \bar{A}=\Omega_{\bar{A}\left[U_{1}^{ \pm 1}, \ldots, U_{d}^{ \pm 1}\right] / \bar{A}}^{\bullet}$

If $A$ is a topological ring, whose topology is $I$-adic for some ideal $I$, then define $q-\Omega_{A\left\langle U_{1}^{ \pm 1}, \ldots, U_{d}^{ \pm 1}\right\rangle / A}^{\bullet}$ to be the $I$-adic completion of $q-\Omega_{A\left[U_{1}^{ \pm 1}, \ldots, U_{d}^{ \pm 1}\right] / A^{\bullet}}^{\bullet}$

Proposition 3.2. If $R=\mathcal{O}\left\langle T_{1}^{ \pm 1}, \ldots, T_{d}^{ \pm 1}\right\rangle$ then $\mathbb{A} \Omega_{R / \mathcal{O}}$ is quasi-isomorphic to the $q$-de Rham complex $q-\Omega_{\mathbb{A}_{\text {inf }}^{\bullet}\left\langle U_{1}^{ \pm 1}, \ldots, U_{d}^{ \pm 1}\right\rangle / \mathbb{A}_{\text {inf }}}$ associated to $q=[\varepsilon] \in \mathbb{A}_{\text {inf }}^{\times}$.

Proof. It follows from the proof of Theorem 3.1, and the general definition of $\mathbb{A} \Omega_{R / \mathcal{O}}$, that in this case $\mathbb{A} \Omega_{R / \mathcal{O}}$ is quasi-isomorphic (and not just almost 
so) to

$$
L \eta_{\mu} R \Gamma_{\text {cont }}\left(\mathbb{Z}_{p}^{d}, H_{\text {proét }}^{0}\left(\widetilde{X}, \mathbb{A}_{\text {inf }, X}\right)\right) .
$$

This can be explicitly calculated and is quasi-isomorphic to

$$
q-\Omega_{\mathbb{A}_{\mathrm{inf}}}^{\bullet}\left\langle U_{1}^{ \pm 1}, \ldots, U_{d}^{ \pm 1}\right\rangle / \mathbb{A}_{\mathrm{inf}}
$$

$\left(U_{i}\right.$ corresponds to the Teichmüller lift of $\left.\left(T_{i}, T_{i}^{1 / p}, T_{i}^{1 / p^{2}}, \ldots\right) \in \widetilde{R}^{b}\right)$.

Our complex $\mathbb{A} \Omega_{\mathfrak{X} / \mathcal{O}}$ may therefore be seen as a natural extension of the $q$-de Rham complex $q-\Omega_{\mathbb{A}_{\text {inf }}^{\bullet}\left\langle U_{1}^{ \pm 1}, \ldots, U_{d}^{ \pm 1}\right\rangle / \mathbb{A}_{\text {inf }}}$, defined initially only for tori $\operatorname{Spf} \mathcal{O}\left\langle T_{1}^{ \pm 1}, \ldots, T_{d}^{ \pm 1}\right\rangle$ with a fixed choice of coordinates, to arbitrary smooth, formal $\mathcal{O}$-schemes $\mathfrak{X}$.

\section{Some examples}

We give an example illustrating the sharpness of our result, namely that it is possible to have more torsion in crystalline cohomology than in étale cohomology.

Although the following does not fall within the scope of our result, as it involves non-smooth stacks, it heuristically explains what can happen:

Example 4.1. Let $G=\mathbb{Z} / p \mathbb{Z}$, and $H=\mu_{p}$, both viewed as finite flat group schemes over $\mathcal{O}$. Choose a map $\eta: G \rightarrow H$ that is an isomorphism on the generic fibre and trivial on the special fibre by using a primitive $p^{\text {th }}$ root of unity in $C$. Let $T \rightarrow B H$ be the universal $H$-torsor and $S=T \times_{B H} B G$ its pullback along $\eta$. Then $S_{C}$ is a point as it is the universal $G$-torsor, while $S_{k} \cong B G_{k} \times \mu_{p}$ is non-reduced. It is then easy to see that the crystalline cohomology of $S_{k}$ has a lot of torsion, while the étale cohomology of $S_{C}$ is trivial.

One can easily push the preceding example into the world of proper smooth schemes:

Example 4.2. Let $p=2$, and fix an Enriques surface $S / \mathcal{O}$ whose special fibre is "singular" in the sense that it has fundamental group $\mathbb{Z} / 2 \mathbb{Z}[3, \S 3]$. Fix also an auxiliary elliptic curve $E / \mathcal{O}$, and choose a map $\mathbb{Z} / 2 \mathbb{Z} \rightarrow E$ that is non-trivial on the generic fibre and trivial on the special fibre. Pushing out the universal cover of $S$ along this map gives an $E$-torsor $D \rightarrow S$ whose generic fibre $D_{C} \rightarrow S_{C}$ is non-split, i.e., does not admit a section, but whose special fibre $D_{k} \rightarrow S_{k}$ is split. 
Thus $D$ is a proper smooth $\mathcal{O}$-scheme of relative dimension 3 and one can show, using the Leray spectral sequence for $D \rightarrow X$, that $H_{\text {ét }}^{2}\left(D_{C}, \mathbb{Z}_{2}\right)$ is torsion-free while $H_{\text {crys }}^{2}\left(D_{k} / W(k)\right)$ has non-trivial 2-torsion.

\section{Acknowledgements}

Bhatt was partially supported by NSF grant DMS 1340424 and both Bhatt and Scholze would like to thank the University of California, Berkeley, and the MSRI for their hospitality during parts of the project. The work was carried out while Morrow was funded by the Hausdorff Center for Mathematics, and Scholze was a Clay Research Fellow. All authors thank the Clay Foundation for funding during parts of the project

\section{References}

[1] P. Berthelot and A. Ogus, Notes on crystalline cohomology. Princeton University Press, Princeton, N.J.; University of Tokyo Press, Tokyo (1978), ISBN 0-691-08218-9.

[2] B. Bhatt, M. Morrow and P. Scholze, Integral p-adic Hodge theory. Preprint arXiv:1602.03148 (2016).

[3] E. Bombieri and D. Mumford, Enriques' classification of surfaces in char p. III. Invent. Math., 35 (1976), 197-232.

[4] X. Caruso, Conjecture de l'inertie modérée de Serre. Invent. Math., 171 (2008), no. 3, 629-699.

[5] P. Deligne, Théorie de Hodge. II. Inst. Hautes Études Sci. Publ. Math., (1971), no. 40, 5-57.

[6] G. Faltings, p-adic Hodge theory. J. Amer. Math. Soc., 1 (1988), no. 1, 255-299.

[7] G. Faltings, Integral crystalline cohomology over very ramified valuation rings. J. Amer. Math. Soc., 12 (1999), no. 1, 117-144.

[8] L. Fargues, Quelques résultats et conjectures concernant la courbe. Astérisque, (2015), no. 369, 325-374. In: De la géométrie algébrique aux formes automorphes (I).

[9] J.-M. Fontaine, Exposé II: Les corps des périodes p-adiques. Avec un appendice par Pierre Colmez: Le nombres algébriques sont denses dans $B_{d R}^{+}$. In: Périodes $p$-adiques. Séminaire du Bures-sur-Yvette, France, 
1988., 59-111, appendix 103-111, Paris: Société Mathématique de France (1994).

[10] R. Huber, Étale cohomology of rigid analytic varieties and adic spaces. Aspects of Mathematics, E30, Friedr. Vieweg \& Sohn, Braunschweig (1996), ISBN 3-528-06794-2.

[11] L. Illusie, Complexe de de Rham-Witt et cohomologie cristalline. Ann. Sci. École Norm. Sup. (4), 12 (1979), no. 4, 501-661.

[12] K.-W. Lan and J. Suh, Vanishing theorems for torsion automorphic sheaves on compact PEL-type Shimura varieties. Duke Math. J., 161 (2012), no. 6, 1113-1170.

[13] A. Langer and T. Zink, De Rham-Witt cohomology for a proper and smooth morphism. J. Inst. Math. Jussieu, 3 (2004), no. 2, 231-314.

[14] P. Scholze, Perfectoid spaces. Publ. Math. Inst. Hautes Études Sci., 116 (2012), 245-313.

[15] P. Scholze, p-adic Hodge theory for rigid-analytic varieties. Forum Math. Pi, 1 (2013) e1, 77.

Department of Mathematics, University of Michigan

2074 East Hall, 530 Church Street, Ann Arbor, MI 48109-1043, USA

E-mail address: bhargav.bhatt@gmail.com

Mathematisches Institut, Universität Bonn

Endenicher Allee 60, 53115 Bonn, Germany

E-mail address: morrow@math.uni-bonn.de

Mathematisches Institut, Universität BonN

Endenicher Allee 60, 53115 Bonn, Germany

E-mail address: scholze@math.uni-bonn.de

Received August 25, 2015 